\title{
Diabetes mellitus tipo 2 e a suscetibilidade de infecção por mycobacterium tuberculosis: apresentação das doenças, resposta e tratamento
}

\author{
Type 2 diabetes mellitus and mycobacterium tuberculosis infection susceptibility: disease \\ presentation, response and treatment
}

\begin{abstract}
Sensibilidad a la infección por diabetes mellitus tipo 2 y mycobacterium tuberculosis: presentación, respuesta y tratamiento de la enfermedad
\end{abstract}

Raissa Freitas Barbosa ${ }^{1 *}$, Fernanda Farias Costa ${ }^{1}$, Karen Vitória Lima Silva ${ }^{1}$, Vitória Estefany Carvalho Dutra $^{1}$, Natália Carvalho Sousa ${ }^{1}$, Esdras Welesson Matias de Sousa ${ }^{1}$, Samyra Gomes Mota ${ }^{1}$, Fabíola Santos Lima de Oliveira², Domingos Magno Santos Pereira1', Cristiane Santos Silva e Silva Figueiredo'.

\section{RESUMO}

Objetivo: Descrever a suscetibilidade de indivíduos com Diabetes tipo 2 (DM2) desenvolverem tuberculose pulmonar ativa, assim como apresentar as doenças, diagnóstico e tratamento. Revisão bibliográfica: Há alguns anos, notou-se a associação entre DM2 e infecções oportunistas causadas por micobactérias, com ênfase no Mycobacterium tuberculosis. Considerando que as duas patologias têm alto índice de mortalidade, a associação entre ambas, causa atemorização à saúde pública. De fato, tal associação leva a piores prognósticos quando comparado somente a indivíduos acometidos pelo DM2. A hiperglicemia traz sérios riscos à saúde dos portadores de DM2, com relatos de que portadores de DM2 têm piores desfechos da tuberculose ativa pelo fato do diagnóstico tardio, mau controle da glicemia, e deficiências no sistema imune ocasionadas pela hiperglicemia o que levam a um tratamento mais conflitante ocasionando maior número de óbitos. Considerações finais: Esta revisão apresenta os fatores que influenciam o acometimento e desenvolvimento de tuberculose pulmonar ativa em indivíduos com DM2, e como se dá o diagnóstico e o melhor tratamento estabelecido até os dias atuais.

Palavras-chave: Diabetes, Infecção, Tuberculose, Sistema imune, Associação.

\section{ABSTRACT}

Objective: To describe the susceptibility of individuals with type 2 diabetes (DM2) to develop active pulmonary tuberculosis, as well as to present the diseases, diagnosis and treatment. Bibliographic review: A few years ago, the association between DM2 and opportunistic infections caused by mycobacteria was noted, with emphasis on Mycobacterium tuberculosis. Considering that both pathologies have a high mortality rate, the association between them causes public health fear. In fact, this association leads to worse prognosis when compared only to individuals affected by DM2. Hyperglycemia poses serious health risks to DM2 patients, with reports that DM2 patients have worse outcomes from active tuberculosis due to late diagnosis, poor blood glucose control, and also deficiencies in the immune system caused by hyperglycemia, thus resulting in a more conflicting treatment, leading to a greater number of deaths. Final considerations: This review presents the factors that influence the onset and development of active pulmonary tuberculosis in individuals with DM2, and how the diagnosis is made and the best treatment established to date.

Keywords: Diabetes, Infection, Tuberculosis, Immune system, Association.

1 Universidade Ceuma (UNICEUMA), Imperatriz - MA. *E-mail: raissabarbosa96a@gmail.com

${ }^{2}$ Faculdade Facimp Wyden, Imperatriz - MA.

SUBMETIDO EM: 10/2021

ACEITO EM: 10/2021

PUBLICADO EM: 10/2021 


\section{RESUMEN}

Objetivo: Describir la susceptibilidad de los individuos con diabetes tipo 2 (DM2) a desarrollar tuberculosis pulmonar activa, así como presentar las enfermedades, diagnóstico y tratamiento. Revisión bibliográfica: Hace unos años se notó la asociación entre DM2 e infecciones oportunistas por micobacterias, con énfasis en Mycobacterium tuberculosis. Considerando que ambas patologías tienen una alta tasa de mortalidad, la asociación entre ellas provoca temor en la salud pública. De hecho, esta asociación conduce a un peor pronóstico si se compara solo con los individuos afectados por DM2. La hiperglucemia presenta serios riesgos para la salud de los pacientes con DM2, con informes de que los pacientes con DM2 tienen peores resultados de la tuberculosis activa debido a un diagnóstico tardío, un control deficiente de la glucosa en sangre y también deficiencias en el sistema inmunológico causadas por la hiperglucemia, que conducen a un tratamiento más conflictivo que conduce a un mayor número de muertes. Consideraciones finales: Esta revisión presenta los factores que influyen en la aparición y desarrollo de la tuberculosis pulmonar activa en individuos con DM2, y cómo se realiza el diagnóstico y el mejor tratamiento establecido hasta la fecha.

Palabras clave: Diabetes, Infección, Tuberculosis, Sistema inmunológico, Asociación.

\section{INTRODUÇÃO}

Diabetes Mellitus (DM) representa um problema de saúde pública de proporções globais. Estima-se que existam 463 milhões de pessoas acometidas mundialmente por essa enfermidade. Segundo a Organização Mundial da Saúde (OMS), a DM caracteriza-se pela elevação da glicose sanguínea, a hiperglicemia, que ocorre pela falha na ação ou na forma de secreção de insulina, hormônio produzido no pâncreas pelas células beta que tem atividade endócrina. Tal desequilíbrio ocasiona o distúrbio metabólico e pode ser potencialmente grave (AMERICAN DIABETES ASSOCIATION (ADA), 2017; INTERNATIONAL DIABETES FEDERATION (IDF), 2019).

Existem diversos tipos de diabetes, os mais discutidos na literatura são: Tipo 1 (DM1), Tipo 2 (DM2), Diabetes Gestacional (DMG), e Monogênicos (MODY 1, 2 e 3), sendo a DM1 e DM2 os mais conhecidos. A DM1 abrange de 5 a $10 \%$ dos indivíduos, sendo mais comum em crianças e adolescentes, e é considerada autoimune sem causa totalmente estabelecida. Já a DM2 é mais diagnosticada nos adultos, no entanto este cenário tem mudado consideravelmente nos últimos anos, afetando também crianças, adolescentes e jovens, representando 90 a 95\% dos casos (IDF, 2019).

A DM2 é o tipo mais comum da doença, classificada como Doença Crônica Não Transmissível (DCNT) que ocorre pela insuficiência da ação insulínica, que é produzida pelas células beta pancreáticas ocasionando resistência à insulina. Quando ocorre a resistência à insulina, o pâncreas começa a produzir mais insulina para tentar estabelecer os níveis plasmáticos adequados no organismo. Assim, quando essa produção entra em exacerbação, ocorre a diabetes, levando à hiperglicemia e glicotoxicidade no organismo (GARDNER DG e GREENSPAN D, 2011).

Estudos mostram que a prevalência da diabetes teve um crescimento avassalador nos últimos 20 anos. Um a cada onze adultos tem diabetes, e 1 a cada 2 adultos ainda não foram diagnosticados com diabetes pela ausência de sintomas ou por negligenciá-los. O crescimento do número de casos tem causado discussões mundo afora, pois é consequência de diferentes fatores como obesidade, histórico familiar, sedentarismo, fatores socioeconômicos, entre outros. A IDF estima que haverá 578 milhões de diabéticos em 2030, e esse número pode chegar a 700 milhões de pessoas diagnosticadas em 2045 (IDF, 2019).

A DM diminui consideravelmente o tempo de vida dos portadores para 10 anos a menos por causar incapacidade e baixa qualidade de vida (NUNES MM, 2020). Em 2019, cerca de 4,2 milhões de pessoas morreram em consequência da doença, sendo que mundialmente, $11,3 \%$ das mortes são por diabetes e suas complicações. A diabetes afeta todas as faixas etárias sem distinção, e dentre os 463 milhões de pessoas 
com diabetes, considera-se que $50,1 \%$ não sabem que são portadores levando a danos inconversíveis, compondo assim uma das maiores causas de incapacidade e morbimortalidade atualmente (SALIN AB, 2019; REIS MAOM, 2021).

Com o passar dos anos e diversos estudos sendo realizados, a ciência mostra que o indivíduo com DM2 em descompensação tem maior facilidade em contrair infecções quando comparado ao indivíduo com a diabetes controlada (MOUTINHO ILD, 2011). Uma das infecções mais recorrentes é a causada pelo Mycobacterium tuberculosis (Mtb) ou Bacilo de Koch (BK), uma bactéria patogênica que leva ao diagnóstico da tuberculose (TB). A TB é uma morbidade descoberta há mais de 150 anos, altamente prevalente e com alto índice de morbidade e mortalidade até os dias atuais, apesar de já existir vacina, diagnóstico e tratamento estabelecido (CENTERS FOR DISEASE CONTROL AND PREVENTION, 2012).

O Mtb afeta o aparelho respiratório, em especial os pulmões, causando a tuberculose pulmonar. A forma de transmissão se dá de pessoa a pessoa, quando ela se encontra na fase ativa da doença, pelo ar, tosse, espirro ou fala (COSTA RR, et al., 2018; BATISTA CP, 2021).

Além disso, a OMS pressupõe que existam 10,4 milhões de casos novos e 1,5 milhão de mortes por TB a cada ano. Associado a DM2 descompensada, o risco aumenta em 8 vezes nos pacientes com a associação dessas patologias. Entre 2009 e 2017, observou-se que o número de indivíduos com DM2 infectados pelo Mtb aumentou de $5,5 \%$ para $7,8 \%$, geralmente na faixa etária entre 50 a 70 anos, manifestando séria preocupação aos órgãos de saúde pública (MINISTÉRIO DA SAÚDE, 2019).

Para diagnosticar a coinfecção, existem alguns exames sensíveis e altamente específicos disponíveis. $O$ diagnóstico de DM2 é feito por exames clínicos e laboratoriais, sendo eles glicemia em jejum e hemoglobina glicosilada, usados tanto para rastreio quanto para diagnóstico da doença. A tuberculose também tem seus métodos baseados em exames clínicos e laboratoriais, considerados padrão ouro para o diagnóstico, sendo eles baciloscopia, cultura de escarro e métodos imaginológicos. Quando há suspeitas de ambas, devem ser feitos em conjunto (COSTA RR, et al., 2018; PETERSMANN A, et al., 2019).

Embora essa problemática esteja em constante estudo, nota-se que há mais de um século há uma elevação em números de casos de indivíduos diabéticos contaminados pela tuberculose pulmonar. Nesse viés, a associação dessas duas patologias é potencialmente grave, gerando altas taxas de mortalidade anualmente, e se considerada a associação delas, esse número deve duplicar nos próximos anos. O presente artigo tem como objetivo apresentar e revisar a suscetibilidade de pacientes com DM2 desenvolverem a tuberculose pulmonar ativa, assim como apresentar as doenças, diagnóstico e tratamento.

\section{REVISÃO BIBLIOGRÁFICA}

\section{Diabetes mellitus tipo 2 e a infecção por mycobacterium tuberculosis}

A DM2 é uma doença que se apresenta pela resistência à insulina ou pela incapacidade de utilizar a insulina produzida pelo organismo, ou seja, o organismo do indivíduo produz insulina, porém esta não completa sua atividade corretamente no organismo, sendo ineficaz. Então a glicose presente na circulação advinda do alimento não é captada, pois os receptores de insulina presentes nas células não funcionam, fazendo com que a glicose não entre na célula. Dessa forma, o pâncreas se esgota e subentende que deve diminuir a produção da insulina, e consequentemente os níveis de glicose sanguínea se elevam, ocasionando hiperglicemia crônica (IDF, 2019). A DM2 possui um grande fator hereditário e relação importante tanto com a obesidade quanto com o sedentarismo (ADA, 2017).

Desse modo a hiperglicemia crônica influencia diretamente na integridade da imunidade dos portadores, de maneira que o sistema imune se altere, provocando diminuição da atividade imunitária, onde as funções leucocitárias ficam limitadas, a quimiotaxia e a fagocitose comprometidas, e levando à deficiência do sistema complemento, diminuição da função neutrofílica e da secreção das citocinas inflamatórias. Com as atividades intracelulares afetadas, há uma maior predisposição do paciente diabético a infecções, dentre elas as infecções do trato respiratório (ALENCAR ACL, 2014). 
A Sociedade Brasileira de Diabetes (SBD) mostra que indivíduos com diabetes mal controlada, ou seja, com dosagem de glicose plasmática superior a $310 \mathrm{mg} / \mathrm{dL}$ e hemoglobina glicosilada $\mathrm{A} 1 \mathrm{c}(\mathrm{HbA} 1 \mathrm{c})$ a $11 \%$ têm maiores chances de desenvolver complicações em vista daqueles com a glicemia inferior a $170 \mathrm{mg} / \mathrm{dL}$ (SBD, 2020).

Desde o início do século XX, é conhecida a associação do DM a infecções fúngicas e bacterianas causadas por patógenos oportunistas, e uma das infecções mais antigas é a Tuberculose (TB) ou tuberculose pulmonar. A tuberculose é uma doença respiratória, infecciosa e transmissível, causada pelo Mycobacterium tuberculosis (Mtb), uma bactéria intracelular facultativa, aeróbia de crescimento lento, pertencente à família Mycobacteriaceae, e de composição extremamente complexa (OLIVEIRA DM, 2013; ABREU RG, et al., 2020).

A TB foi considerada a principal causa de morte por doença infectocontagiosa em 2015 no mundo, ultrapassando o Vírus da Imunodeficiência Humana (HIV). Estima-se que cerca de 1,7 bilhão de pessoas, equivalente a $23 \%$ da população mundial, tenha TB latente, aumentando o risco de desenvolver TB ativa no futuro (SILVA DR, et al., 2018; SANTOS FJ, et al., 2020; MASSABNI AC e BONINI EH, 2019).

A estrutura complexa da parede celular do Mtb confere importantes fatores de virulência. O microrganismo tem uma espessa camada de ácidos micólicos que formam uma barreira hidrofóbica e, embora dificulte a entrada de nutrientes, confere resistência celular e a antibióticos e a resposta imune (SANTOS FJ, et al., 2020). O bacilo (Bacilo de Koch) é capaz de se multiplicar no interior dos macrófagos alveolares, afetando exclusivamente a espécie humana, tendo como alvo os pulmões, em especial as vias aéreas inferiores. $O$ microrganismo se propaga pela via aérea, através de uma pessoa com infecção na fase ativa da doença (bacilífera) (OLIVEIRA DM, 2013).

Indivíduos imunossuprimidos e em desequilíbrio metabólico, como no caso dos portadores de DM2, estão mais expostos e podem ser mais afetados pelo patógeno devido ao seu sistema imunológico não estar tão atuante. Com isso, suas funções inatas e adaptativas são comprometidas e, assim, não conseguem sanar o processo infeccioso, levando à manifestação da doença, passando da fase de latência para sua forma ativa (BANULS AL, et al., 2015; MCMURRY HS, et al., 2019).

Sabe-se que a DM2 aumenta o risco de infecções do trato respiratório inferior pela disfunção dos neutrófilos que acabam por comprometer a imunidade mediada por células. Em pacientes com DM2 descompensada, a disfunção da imunidade celular inata se predispõe a maior virulência do patógeno, e a alta concentração de glicose desempenha maior influência no acometimento da infecção (PIZZOL D, et al., 2016). A inflamação crônica gerada pela obesidade e pelo DM pode estar entrelaçada na suscetibilidade aumentada à TB.

A inflamação crônica de baixo grau agregada ao DM2 gera um quadro inflamatório, sendo considerado um dos fatores cruciais que influenciam no risco de desenvolver TB em pacientes com DM2. Além disso, tem sido demonstrado que a produção de citocinas é diminuída, como a IFN- $\gamma$, em pacientes com DM2 descompensada, citocina esta que é responsável pelas funções efetoras dos macrófagos, permitindo um risco maior de TB ativa, além de possibilitar a falha no tratamento e o aumento de óbitos nos casos da coinfecção (SALGADO BM, et al., 2018).

Ferlita S, et al. (2019) mostra em seu estudo que, em análise feita no ano de 2012, cerca de $50 \%$ dos pacientes diagnosticados com TB eram diabéticos ou pré-diabéticos, o que corrobora com outros trabalhos, como um estudo feito no México que demonstra que 35\% dos pacientes positivos para TB eram diabéticos. A partir disso, observa-se que há relação entre o aumento da incidência de DM2 em países onde a TB também é endêmica (VRIELING F, 2020; WILKINSON RJ, 2021).

A TB ativa costuma evoluir com mais frequência em pacientes com controle glicêmico superior ao desejável (SILVA DR, et al., 2018). Em estudo feito por Pizzol D, et al., (2016) com pacientes portadores de DM2, mostrou-se que o risco de tuberculose ativa era três vezes maior entre aqueles com nível de hemoglobina glicosilada $\mathrm{A} 1 \mathrm{c}(\mathrm{HbA} 1 \mathrm{c}) \geq 7 \%$ do que naqueles com nível de $\mathrm{HbA} 1 \mathrm{c}<7 \%$. 
A hiperglicemia crônica é o fator primário que ocasiona inúmeras complicações fisiológicas e metabólicas da DM (FERREIRA LT, et al., 2011). Indivíduos com descontrole glicêmico estão mais suscetíveis a avançar do quadro de tuberculose latente para tuberculose ativa. Em estudo realizado pela Philadelfia Diabetic Survey, demonstrou-se que a chance de diabéticos dependentes de insulina desenvolverem tuberculose é duas vezes maior do que aqueles não dependentes ou que usam doses baixas de insulina (SILVA DR, et al., 2018).

Bobadilla DVM, et al., (2021) expõem que indivíduos com DM2 têm um risco de 3 a 6,8 vezes maior de desenvolver TB e, consequentemente, ter manifestações mais graves devido à hiperglicemia do que aqueles sem DM2. Na Figura 1 tem-se um exemplo de como o DM2 influencia no desenvolvimento da infecção.

Figura 1 - Fatores que facilitam a infecção por Mycobacterium tuberculosis em diabéticos do tipo 2.

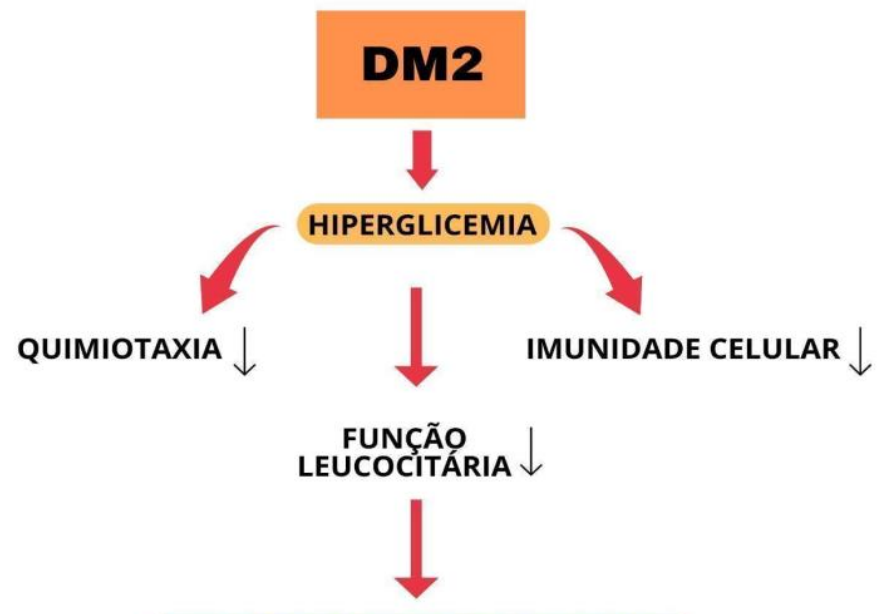

INFECÇÃO DO TRATO RESPIRATÓRIO

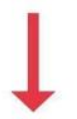

Mycobacterium tuberculosis

Nota: Imagem produzida na plataforma Canva®.

Fonte: Barbosa RF, et al., 2021; baseado em: Nascimento CV e Soares SM, 2019, Ferreira LT, et al., 2011, PIZZOL D, et al., 2016.

Nascimento CV e Soares SM (2019) afirmam que a hiperglicemia causa impacto no controle da TB, aumentando o risco da infecção e quadro de tuberculose ativa, reflexo de um sistema imunológico comprometido. No Brasil, os indivíduos do sexo masculino são os mais afetados, totalizando 80 a $85 \%$ dos acometidos, porém dependendo da região, ocorrem variações no perfil de gênero. No México, por exemplo, os estudos apontaram que o sexo feminino é mais afetado em cerca de $70,4 \%$ em comparação ao sexo masculino, $64,5 \%$. Independente do gênero, a tuberculose deve ser diagnosticada previamente em indivíduos com DM2.

\section{Sintomatologia}

As manifestações clínicas de ambas as doenças dependem das respostas individuais, imunológicas e metabólicas de cada uma. A sintomatologia característica de DM2 é clássica, porém não se manifesta no início da doença. Na maioria dos casos, os indivíduos costumam ser assintomáticos nos primeiros períodos, pois as manifestações clínicas são notadas em períodos em que estes têm o sistema imunológico comprometido pelo quadro hiperglicêmico e, então, as manifestações aparecem, sendo elas sede excessiva, boca seca, alta frequência urinária, indisposição, cansaço, feridas de cicatrização lenta, infecções recorrentes, visão turva, formigamento ou dormência nas mãos e pés (IDF, 2019). 
Em relação à TB, indivíduos infectados e na fase ativa da doença geralmente têm uma queda significativa na imunidade devido a hiperglicemia, sendo assintomáticos no início da infecção, pois a manifestação da tuberculose pulmonar é considerada lenta em comparação à infecção respiratória causada pelo SARS-COV2, que leva de 2 a 14 dias para a manifestação dos sintomas. A TB tem um período longo de latência entre a infecção inicial e a apresentação clínica (XAVIER AR, et al., 2020).

Quando a TB progride da fase de latência para a fase ativa em indivíduos com DM2, estes apresentam um descontrole metabólico significativo, em que o controle glicêmico se torna difícil, ocasionando sérias complicações. Os principais sintomas relatados entre os pacientes são tosse persistente, cansaço intenso, perda de peso, febre, dispneia e suores noturnos, sendo bastante variável de paciente a paciente. Raramente, as equipes médicas relacionam os sintomas a uma coinfecção entre DM2-TB (PIZZOL D, et al., 2016).

\section{Diagnóstico}

O diagnóstico das patologias foi estabelecido há mais de duas décadas e deve ser feito precocemente para melhor conduta clínica. O quadro colaborativo de ações da OMS para a TB e a DM atualmente recomenda que seja feita uma triagem dupla para DM em todos os pacientes com TB e vice-versa. Os exames utilizados para diagnóstico são uma consolidação entre teste de glicemia em jejum, hemoglobina glicada, Pesquisa do Bacilo Álcool-Ácido Resistente (BAAR), cultura de escarro e exames de imagem, servindo tanto para o diagnóstico inicial quanto para acompanhamento do tratamento (SILVA DR, et al., 2018).

\section{Teste de glicemia em jejum e hemoglobina glicosilada}

A glicose plasmática dosada em jejum é o melhor método utilizado para quantificar e relatar as concentrações de glicose presentes no sangue de um indivíduo diabético. Com a glicose alterada, o indivíduo deve ser submetido ao teste de hemoglobina glicada para melhor mapeamento dos índices glicêmicos presentes em seu metabolismo (PIZZOL D, et al., 2016).

Outro método importante muito utilizado no diagnóstico de DM2 desde 2010, juntamente com a glicemia em jejum, é a Hemoglobina glicosilada ( $\mathrm{HbA1c}$ ), um exame preciso que mostra a média glicêmica do paciente no que diz respeito aos 3 meses anteriores à coleta. Em pacientes já diagnosticados com diabetes, o exame deve ser realizado no mínimo duas vezes por ano para melhor controle da glicemia. A dosagem é considerada padrão ouro no diagnóstico de DM2 por refletir tão precisamente os níveis glicêmicos presentes na corrente sanguínea do portador. Em pacientes com DM2 e tuberculose ativa, essa dosagem vem sendo utilizada para melhor acompanhamento do tratamento e possível regressão da doença (SILVA CP e FREITAS DT, 2019).

\section{BAAR e cultura de escarro}

A baciloscopia consiste na observação e contagem de bacilos presentes na amostra de escarro para posterior identificação de Mtb, e configura a técnica mais utilizada mundialmente, por ser de baixo custo, rápida, segura e simples. Após coloração específica, permite-se a visualização do bacilo e, assim, o diagnóstico da TB em 60 a 80\% dos casos (COSTA RR, et al., 2018; SANTOS FJ, et al., 2020). O BAAR apresenta a morfologia do Mtb, com bastonetes delgados e finos, corados em vermelho, sobre fundo corado em azul claro (SILVA CP e FREITAS DT, 2019).

A amostra de escarro mais adequada para o diagnóstico da TB é proveniente da árvore brônquica, adquirida por expectoração. A árvore brônquica é estéril, mas ao fazer a expectoração, o escarro acaba sendo contaminado ao passar pelo trato respiratório inferior, o que consequentemente trará resultados na pesquisa de BAAR (COSTA RR, et al., 2018; SILVA CP e FREITAS DT, 2019; SANTOS FJ, et al., 2020). Para posterior confirmação do BAAR, mesmo em amostra negativa, deve ser feita a cultura do escarro pois a cultura apresenta especificidade de $98 \%$ e sensibilidade de $80 \%$, sendo considerada padrão ouro para o diagnóstico da TB (SILVA DR, et al., 2021).

Em comparação com pacientes sem DM2, a carga bacilar no escarro é maior em pacientes com DM2, os quais também levam mais tempo para a apresentação da cultura. No entanto, as taxas de conversão de cultura de escarro após os dois primeiros meses de tratamento têm semelhança entre pacientes com DM2 e 
sem DM2 (SILVA DR, et al., 2018; COSTA RR, et al., 2018). Após o término dos exames, as amostras devem ser autoclavadas e descartadas em recipientes adequados, por oferecer alto risco biológico à sociedade (SANTOS HP, et al., 2019).

\section{Xpert MTB/RIF e Xpert MTB/RIF Ultra}

Segundo a OMS, algumas lacunas no diagnóstico da tuberculose têm sido detectadas, sendo necessário testes específicos, sensíveis e rápidos. Diante desta necessidade, tem-se, desde 2011, o ensaio Xpert MTB/RIF, um teste baseado na Reação em Cadeia da Polimerase (PCR) em tempo real, que detecta a presença do Mtb através do seu material genético, fazendo o diagnóstico rápido da TB. O teste detecta também as cepas resistentes à rifampicina, um dos medicamentos de primeira escolha para o tratamento (ZHANG M, et al., 2020). O Xpert MTB/RIF é um teste molecular onde tem-se o resultado em apenas 2 horas, sendo mais confortável para o paciente pelo mínimo tempo de espera e requer apenas uma amostra de escarro (ZHANG M, et al., 2020; SILVA DR, et al., 2021).

A partir de modificações do Xpert MTB/RIF, há atualmente o Xpert MTB/RIF ULTRA, que tem mais sensibilidade em cultura em meios líquidos, porém é menos específico que o método anterior. Nesta técnica, os resultados são liberados como negativo, positivo e traços (SILVA DR, et al., 2021). A expectativa é que, a médio prazo, esses testes moleculares possam substituir a baciloscopia (HORNE DJ, et al., 2019).

\section{Raio-X de tórax e tomografia computadorizada}

O exame radiológico e laboratorial, juntamente com a história clínica do paciente, é de extrema relevância em pacientes com diagnóstico de DM2 e TB (CHENG J, et al., 2017). Assim, os achados radiográficos mais encontrados nesses pacientes são opacidades parenquimatosas, segmentares ou lobares, linfonodomegalias hilares ou mediastinais, síndrome do lobo médio, nódulos agrupados, opacidades heterogêneas segmentares ou lobares, nódulos com tamanho de 1 a $3 \mathrm{~cm}$ de diâmetro, cavidades com paredes espessas e disseminação broncogênica e áreas de extensa fibrose. É possível ainda encontrar o infiltrado multinodular acima ou atrás da clavícula, caracterizando um achado característico de TB ativa (SILVA DR, et al., 2021).

Embora o raio-X seja extremamente importante para o diagnóstico da TB, a Tomografia Computadorizada tem sido bastante sensível e específica no diagnóstico pelo fato de mostrar alterações iniciais imperceptíveis pelo raio-X, sendo utilizada em pacientes cujas suspeitas ainda não foram totalmente estabelecidas ou onde o raio-X não foi esclarecedor, e para melhor detalhamento do mediastino (SILVA DR, et al., 2021).

\section{Tratamento}

O tratamento da coinfecção deve ser feito usando metodologias previamente estabelecidas. Os fármacos mais utilizados para tratamento da TB pulmonar são a isoniazida, rifampicina, etambutol e pirazinamida, considerados tratamento de primeira linha, e devem ser administrados durante 6 meses após diagnóstico definitivo. Dado o início do tratamento farmacológico e mudança nos hábitos de vida, em cerca de 2 a 3 meses o controle glicêmico do paciente é normalizado (OLIVEIRA DM, 2013).

Além disso, pacientes com tuberculose com controle inadequado da glicemia têm maiores chances de falha no tratamento, recaída e morte em relação àqueles que mantêm os índices glicêmicos controlados. $O$ controle da glicemia deve ser a prioridade dos médicos em pacientes acometidos por ambas as doenças. Silva DR, et al. (2018) demonstram que pacientes com DM2 apresentam um risco 3,9 vezes maior de falha no tratamento.

Desta maneira, o tratamento da hiperglicemia crônica em indivíduos com DM2 tem como estratégia um controle rigoroso do metabolismo, e melhora dos hábitos de vida como alimentação e atividade física regular, podendo reverter a baixa ação das funções imunológicas e reduzir a suscetibilidade a infecções (ALENCAR ACL, 2014; PIZZOL D, et al., 2016). Ressalta-se que a conduta terapêutica da tuberculose tem efeitos hiperglicemiantes, dificultando o controle metabólico do paciente com diabetes, visto que o DM2 altera a farmacocinética dos medicamentos utilizados para tratamento da tuberculose, contribuindo para o controle adequado do paciente diabético (ALENCAR ACL, 2014). 


\section{CONSIDERAÇÕES FINAIS}

Visto que a frequência de tuberculose ativa é mais comum em diabéticos do tipo 2 com descompensação glicêmica e sistema imune comprometido, podendo resultar em sérias complicações para 0 paciente acometido sem diagnóstico e tratamento prévio. É de extrema importância o desenvolvimento de práticas que visam a promoção de rotinas para o conhecimento das doenças e o autocuidado, como uma forma de prevenir o risco de novos casos e as possíveis complicações atreladas a essa correlação. Dado o exposto entende-se que o paciente diabético deve manter a glicemia controlada através da alimentação adequada, atribuir a prática de exercícios físicos ao seu dia a dia, receber o tratamento farmacológico correto, evitando assim queda no sistema imune deixando-os suscetíveis a infecção.

\section{REFERÊNCIAS}

1. ABREU RG, et al. Tuberculose e diabetes: associação com características sociodemográficas e de diagnóstico e tratamento. Brasil, 2007-2011. Revista Brasileira de Epidemiologia, 2020; 23(1): 1-13.

2. ALENCAR ACL. Tuberculose e diabetes mellitus: abordagens na trajetória espaço-temporal. 2014. Dissertação (Mestrado em Ciências) - Escola de Enfermagem, Universidade de São Paulo. São Paulo, 2014; 145 p.

3. AMERICAN DIABETES ASSOCIATION. Standards of medical care in diabetes. Diabetes Care. 2017; 40(1): 1-131.

4. BANULS AL, et al. Mycobacterium tuberculosis: ecology and evolution of a human bacterium. Journal of medical microbiology, 2015; 64(11): 1261-1269.

5. BATISTA CP. A epidemiologia da tuberculose humana no mundo. Revista Científica Fesa, 2021; 1(2): $19-37$.

6. BOBADILLA DVM, et al. Mycobacterial Growth Inhibition Assay (MGIA) as a host directed diagnostic tool for the evaluation of the immune response in subjects living with type 2 diabetes mellitus. Frontiers in Cellular and Infection Microbiology, 2021; 18(11): 640-707.

7. CENTERS FOR DISEASE CONTROL AND PREVENTION (CDC). Centers for Disease Control and Prevention. Tuberculosis (TB). 2012. Disponível em: https://www.cdc.gov/tb USA 2012. Acesso em: 14 mar. 2021.

8. CHENG J, et al. Mutual impact of diabetes mellitus and tuberculosis in China. Biomedical and Environmental Sciences, 2017; 30(5): 384-389.

9. COSTA RR, et al. Diagnóstico laboratorial da tuberculose: Revisão de literatura. Revista Médica de Minas Gerais, 2018; 28(5): 280-525.

10. FERLITA S, et al. Diabetes mellitus tipo 2 e sistema imunológico alterado levando à suscetibilidade a patógenos, especialmente Mycobacterium tuberculosis. Journal of Clinical Medicine, 2019; 8(12): 22-19.

11. FERREIRA LT, et al. Diabetes melito: hiperglicemia crônica e suas complicações. Arquivos Brasileiros de Ciências da Saúde, 2011; 36(3): 182-188.

12. GARDNER DG, GREENSPAN D. Chapter 17. Greenspan's Basic \& Clinical Endocrinology, McGraw-Hill Medical, New York, NY, USA, 2011.

13. HORNE DJ, et al. Xpert MTB/RIF and Xpert MTB/RIF Ultra for pulmonary tuberculosis and rifampicin resistance in adults. Cochrane Database of Systematic Reviews, 2019; 6(6): 1-279.

14. INTERNATIONAL DIABETES FEDERATION (IDF). Atlas de Diabetes da IDF, 9 edição 2019. Disponível em: https://www.diabetesatlas.org/data/en/world/ Acesso em: 13 mar. 2021.

15. MASSABNI AC, BONINI EH. Tuberculose: história e evolução dos tratamentos da doença. Revista Brasileira Multidisciplinar, 2019; 22(2): 6-34.

16. MINISTÉRIO DA SAÚDE. Secretaria de Vigilância em Saúde. Manual de recomendações para o controle da tuberculose no Brasil. $\quad$ Brasil, $2019 . \quad$ Disponível em: https://bvsms.saude.gov.br/bvs/publicacoes/manual_recomendacoes_controle_tuberculose_brasil_2_ed.pdf. Acesso em: 14 mar. 2021.

17. MCMURRY HS, et al. Coprevalência de diabetes mellitus tipo 2 e tuberculose em países de baixa e média renda: uma revisão sistemática. Pesquisa e Revisões sobre Diabetes/Metabolismo, 2019; 35(1): 30-66.

18. MOUTINHO ILD. Tuberculose: aspectos imunológicos na infecção e na doença. Revista Médica de Minas Gerais, $2011 ; 21(1): 42-48$.

19. NASCIMENTO CV, SOARES SM. Manejo integrado de tuberculose e diabetes: uma revisão integrativa. Revista Panamericana de Salud Pública, 2019; 43(1): 1-9.

20. NUNES MM, et al. Percepção de enfermeiros sobre o autocuidado frente às complicações do diabetes na qualidade de vida. Research, Society and Development, 2020; 9(11): p. e80891110645-e80891110645.

21. OLIVEIRA DM. Mycobacterium tuberculosis e a resistência do bacilo de Koch. 2013. Tese (Doutorado em Ciências da Saúde) - Universidade Fernando Pessoa, Porto. 2013; 53 p. 
22. PETERSMANN A, et al. Definition, classification and diagnosis of diabetes mellitus. Experimental and Clinical Endocrinology \& Diabetes, 2019; 127(1): S1-S7.

23. PIZZOL D, et al. Tuberculosis and diabetes: current state and future perspectives. Tropical Medicine and International Health, 2016; 21(6): 694-702.

24. REIS MAOM, et al. Complicações cardiovasculares em pacientes com Diabetes Mellitus Tipo Revista Eletrônica Acervo Saúde, 2021; 13(3): 6426.

25. SALGADO BM, et al. Metabolomics applied to the discovery of tuberculosis and diabetes mellitus biomarkers. Biomarkers in Medicine, 2018; 12(9): 1001-1013.

26. SALIN AB, et al. Diabetes Mellitus tipo 2: perfil populacional e fatores associados à adesão terapêutica em Unidades Básicas de Saúde em Porto Velho-RO. Revista Eletrônica Acervo Saúde, 2019; (33): 1257.

27. SANTOS HP, et al. A importância da biossegurança no laboratório clínico de biomedicina. Revista Saúde em Foco, $2019 ; 11(1): 210-255$.

28. SANTOS FJ, et al. Manual técnico de investigação laboratorial de tuberculose. Rio de Janeiro: Fundação Oswaldo Cruz; 2020. $55 \mathrm{p}$.

29. SILVA CP, FREITAS DT. Tuberculose pulmonar: estudo de caso. 2019.

30. SILVA DR, et al. Consenso sobre o diagnóstico da tuberculose da Sociedade Brasileira de Pneumologia e Tisiologia. Jornal Brasileiro de Pneumologia, 2021; 47(1): 1-13.

31. SILVA DR, et al. Fatores de risco para tuberculose: diabetes, tabagismo, álcool e uso de outras drogas. Jornal Brasileiro de Pneumologia, 2018; 44(2): 145-152.

32. SOCIEDADE BRASILEIRA DE DIABETES (SBD). Diretrizes Sociedade Brasileira de Diabetes 2019-2020. 2020. Disponível em: http://www.saude.ba.gov.br/wp-content/uploads/2020/02/Diretrizes-Sociedade-Brasileira-de-Diabetes2019-2020.pdf. Acesso em: 19 mai. 2021.

33. VRIELING F. Tuberculosis and type 2 diabetes. 2020. Tese (Doutorado em Ciências) - Leiden University, Leiden, 2020; $253 \mathrm{p}$.

34. WILKINSON RJ. Tuberculosis and type 2 diabetes mellitus: an inflammatory danger signal in the time of coronavirus disease 2019. 2021; 72(1): 79-81.

35. XAVIER AR, et al. COVID-19: manifestações clínicas e laboratoriais na infecção pelo novo coronavírus. Jornal Brasileiro de Patologia e Medicina Laboratorial, 2020; 56(1): 1-9.

36. ZHANG M, et al. Diagnostic accuracy of the new Xpert MTB/RIF Ultra for tuberculosis disease: A preliminary systematic review and meta-analysis. International Journal of Infectious Diseases, 2020; 90(1): 35-45. 